\title{
Recognition of Leaves in Videos
}

\author{
Naveena M, Vidyashankara, G Hemantha Kumar
}

\begin{abstract}
The aim of this paper work is to design a user independent framework for recognizing and classifying the leaves in a video frames. This project involves classification of leaves using KNN (K- Nearest Neighbor) as a classifier. SURF (Speeded-Up Robust Features) and LBP (Local Binary Pattern) features are used for extracting Scale, Orientation etc., In the first step our proposed model can extract most distinguish key-frames and then from extracted key-frames it detects the leaf color and recognize the different class of leaves.

Keywords: Graph Cu, Segmentation, Recognition.
\end{abstract}

\section{INTRODUCTION}

Plants play an important role in human life by providing shelter and by maintaining a healthy breathable environment. There are huge numbers of plant species worldwide to handle such volumes of information creating a plant database for quick and efficient classification and recognition method has become an area of active research. People recognize a specific plant type by characteristics of its leaf shape, color, texture etc., Environment and the climate are very much dependent on plants.

Natural resource managers especially those interested in wildlife management must be able to evaluate the presence or absence of many plant species. It involves innovation method of plant recognition system using digital videos of plant leaves due to lack of awareness of plant knowledge. The plant categories are becoming rare and many plants are about to extinct, because of this there is a need for easy and efficient recognition and classification of plant by their category. The leaves videos of a particular species are collected and stored in the database, then the input video is converted into key frames. In that key frames we can find the important features that are useful for classification of various plant species such as, Color, Shape, textures and structure of leaf. The main aim of this project is to concentrate on the plant classification based on the global feature of the leaf.

\section{LITERATURE REVIEW}

Below are the some of the research papers that have already worked regarding the proposed system:

Research article KADIR, et al [1] proposed a method that incorporate shape, color, and texture feature. They have used probabilistic neural networks (PNN) as a classifier for the plant leaf classification. In this case color also playing important role in identification process.

Revised Manuscript Received on February 10, 2020

*Correspondence Author

Naveena M*, Scientific Officer, University of Mysore, Mysore, Karnataka, India. scientificofficer@uni-mysore.ac.in

Vidyashankara, Research Scholar, University of Mysore, Mysore, Karnataka, India.

G Hemantha Kumar, Vice Chancellor, University of Mysore, Mysore, Karnataka, India.ghk.2007@yahoo.com

(c) The Authors. Published by Blue Eyes Intelligence Engineering and Sciences Publication (BEIESP). This is an open access article under the CC BY-NC-ND license (http://creativecommons.org/licenses/by-nc-nd/4.0/)

According to Rashad, et al [2] introduced a novel approach for classification of plants. They have utilized a combined classifier learning vector quantization along with the basic function. One can classify a plant having only a portion available that is in itself enough as the proposed system requires only color feature. This system can be useful for the researchers of medicinal plant.

As per the research article introduced by Arun, et al [3] presented an automated system for recognizing the medicinal plant leaves .The features include SURF and HSV color feature extraction. Six different classifiers are used to classify the plant leaves based on feature values.

\section{PROPOSED METHODOLOGY}

We have proposed a model to recognize the leaves based on SURF (Speeded Up Robust Features) and LBP (Local Binary Patterns) features by loading the samples which are stored in the directory as segmented frames, video sequence is taken as input from the camera and for each segmented key frames of input video leaf region are detected and recognized and also classification was done by the proposed method.

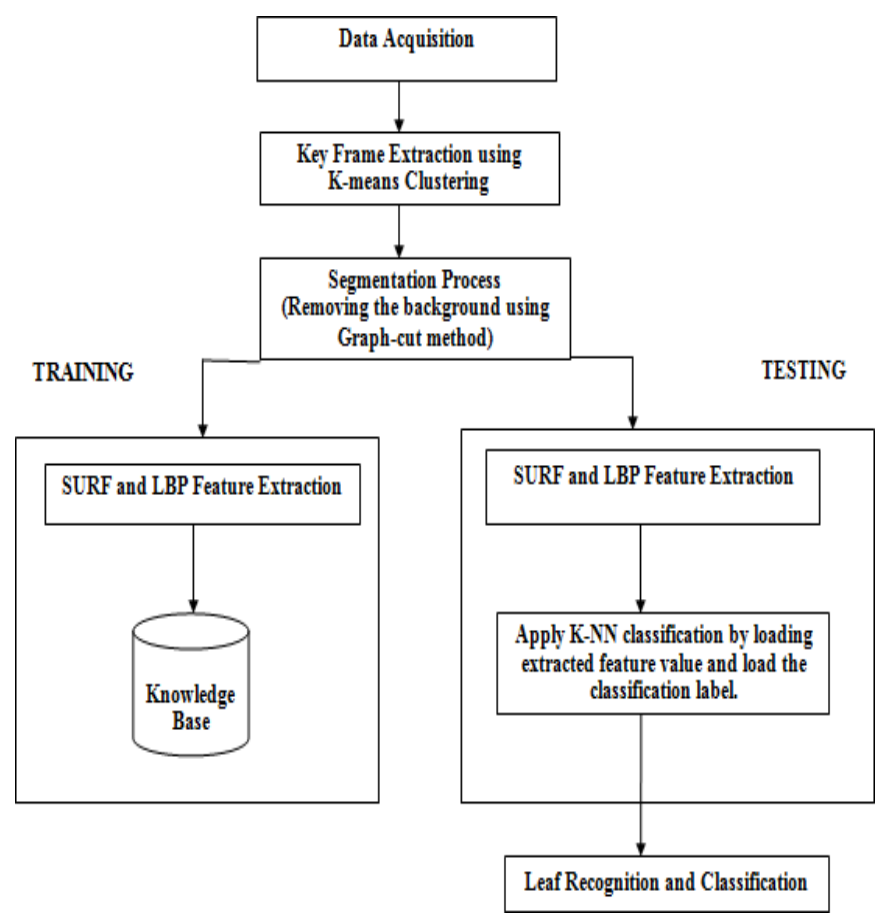

Fig 1: Architecture of Proposed System

\section{DIFFERENT MODULES IN PROPOSED SYSTEM}

Contains different leaves with similar and different leave projection. Data samples are subjected to necessary pre-processing techniques.

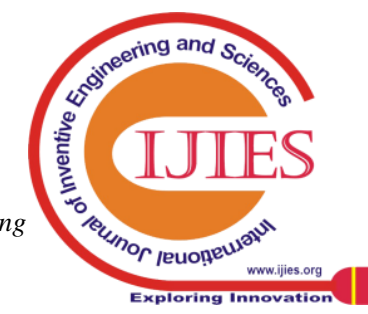




\section{Recognition of Leaves in Videos}

Most important step in pre-processing is the key frame extraction in which only the significant frames selected as key frame and it is converted to leaf segmented frames while removing the background.

\subsection{Data Acquisition and Pre- Processing Stage}

Pre-Processing is the initial stage of leaf classification. In this work, sample videos are acquired by digital camera. A data sample Feature Extraction involves extracting the feature values from the input images.

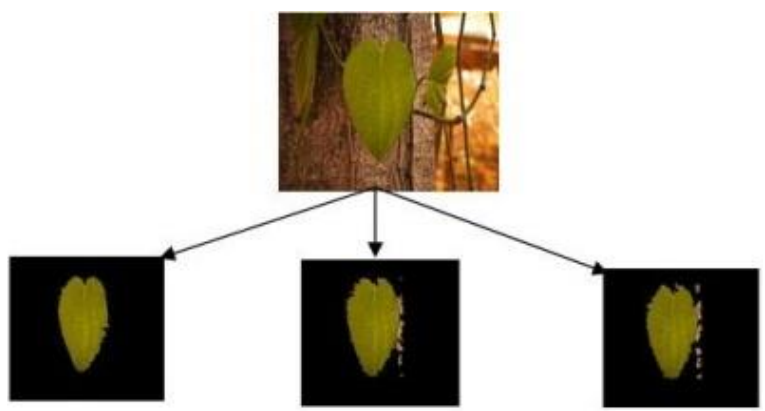

Fig 2: Showing Segmentation using Graph-Cut Method

\subsection{Segmentation}

The purpose of image segmentation is to partition an image into meaningful regions with respect to a particular application.

In our proposed method we have used Graph-Cut similarity Detection based Segmentation Approach .Graph-Cut Segmentation is based on the graph partitioning methods where an image is considered as an undirected graph whose pixels represents the nodes and the distance between neighboring pixels forms an edge. Each edge is given a weight and those weight vectors are used as the segmentation parameters. It holds various advantages like accuracy and performance, Graph cut method can be applied in textured images, noisy images, and color images.

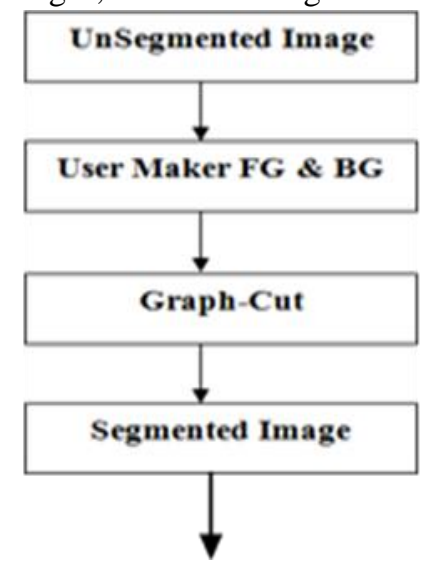

\subsection{Feature Extraction Method}

\subsubsection{Surf Feature Extraction.}

In computer vision, Speeded Up Robust Features (SURF) is a global feature detector and descriptor. The SURF is several times faster than Scale-Invariant transfer Feature (SIFT). To detect the interested points, SURF uses an integer approximation of blob detector. This detection should be possible when the image shows the object with different transformation. This includes the parameters like count, location, scale and orientation.

\subsubsection{Local Binary Patterns (LBP) Feature}

Local Binary Pattern features (LBP) is a simple and efficient texture extraction method that which is used to label the pixels of an image by thresholding, the neighborhood of each pixel and provides the results as a binary number.

\subsection{Classification}

Classification typically employs two phases of processing that is training and testing. Classification is the process by which we attribute a class label to set of measurements. The K-Nearest Neighbor rule achieves consistently high performance without a priori assumptions about the distributions from which the training examples are drawn .The K- NN classifier extends this idea by taking the KNearest points and assigning the majority value

.K-NN classification using an instance-based classifier can be a simple matter of locating the nearest neighbor in instance space and labeling the unknown instance with the same class label as that of the known neighbor. This approach is often referred to as a nearest neighbor classifier.

The K-NN classifier extends this idea by taking the $\mathrm{k}$-nearest points and assigning the value of the majority. It is common to select $\mathrm{k}$ small and $\mathrm{k}$ large values which help to reduce the effects of noisy points within the training dataset. And the choice of $\mathrm{k}$ is often performed through cross validation. The Euclidean distance can be defined as $\mathrm{D}$,

$I I A-B I I=\sqrt{I I A I I^{2}+I I B I I^{2}-2 * A \cdot B}$

Where $\mathrm{D}$ is a Euclidean Distance between $\mathrm{A}$ and $\mathrm{B}$.
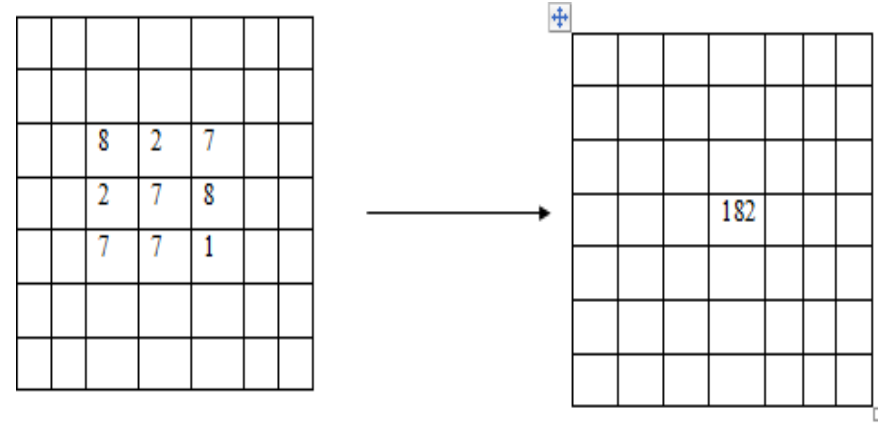

$\begin{array}{llllllll}2^{7} & 2^{\circ} & 2^{3} & 2^{4} & 2^{3} & 2^{2} & 2^{3} & 2^{\circ}\end{array}$

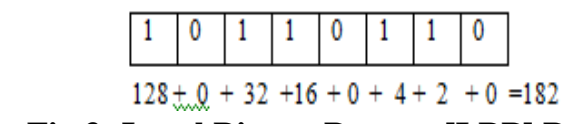

Fig 3: Local Binary Pattern [LBP] Process

\section{DATA SET}

We have collected primary data set. Our dataset consists of 8 different leaves videos. Each class consists of 5 samples. Totally we have 40 sample videos from 8 classes of different leaves. The distance between camera and the focusing object was $10 \mathrm{~cm}$. The duration of video was 10 seconds, with the same duration and same distances we have captured the different classes of leaves video. 
Table 1: Table showing Collected Dataset Information

\begin{tabular}{|c|c|c|c|}
\hline Sl.No & Class Name & No. of Sample & $\begin{array}{l}\text { No. Of. Extracted } \\
\text { Keyframes }\end{array}$ \\
\hline 1 & Class 1 & 5 & 20 \\
\hline 2 & Class 2 & 5 & 30 \\
\hline 3 & Class 3 & 5 & 20 \\
\hline 4 & Class 4 & 5 & 15 \\
\hline 5 & Class 5 & 5 & 12 \\
\hline 6 & Class 6 & 5 & 13 \\
\hline 7 & Class 7 & 5 & 12 \\
\hline 8 & Class 8 & 5 & 11 \\
\hline Total & 8 Classes & 40 & 133 \\
\hline
\end{tabular}

\section{EXPERIMENTAL RESULTS}

Table 2: Table showing Performance of a System

\begin{tabular}{|c|c|}
\hline $\begin{array}{c}\text { Cases Training-Testing } \\
\text { set }\end{array}$ & Accuracy \\
\hline $30-70$ & 50.123 \\
\hline $20-80$ & 40.33 \\
\hline $60-40$ & 64.73 \\
\hline $70-30$ & 56.75 \\
\hline $50-50$ & 64.0625 \\
\hline $40-60$ & 54.5455 \\
\hline $80-20$ & 65.25 \\
\hline
\end{tabular}

We have also performed experiments on different Training and Testing process. The results of $70 \%$ accuracy rate have been achieved.

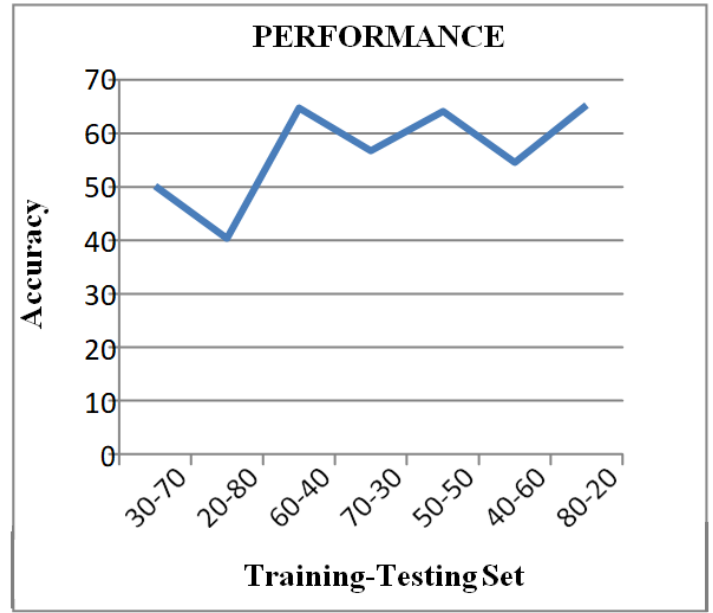

Fig 4: Representing Performance of a System

\section{CONCLUSION}

Recognition of leaf in a video is a very difficult and challenging problem. Most of the previous research work has focused on image of the leaf for recognition and classification with constant background. But in this project we are focused on classification of leaves in videos with varied background. We have tried to implement a system that recognizes leaf in a video by extracting SURF and LBP features. Classification can be done using K-NN as a classifier.

\section{REFERENCES}

1. M.Z.Rashad, S.Khawasik,"Plants Images classification based on textural features using combined classifier", International Journal of Computer Science and Information Technology(IJCSIT),VOL 3,No.4
2. Abdul Kadir, Paulus Insap Santosa,'Leaf Classification using shape,color, and texture", International Journal of Computer Trends and Technology (IJCIT) July-August 2011,PP,225- 230

3. T. Beghin,J.S. Cope,"Shape and Texture Based plant leaf Classification", Advanced Concepts for Intelligent Vision Systems.

4. J.S. Cope, S. Barman," Plant texture classification" using gabor co-occurrences", In advances in visual computing.

5. Mohamed Elhadi Rahmani, Mohamed Reda Hamou, Abdelmalek Amine," Plant Leaves Classification", GecoDe Laboratory Department of Computer Science University of Dr. Tohar Moulay Saida.

6. Chitade,S. Katiyar,"Color Based Image Segmentation using K-means clustering”, International Journal of Engineering Science and Technology, VOL.2 ,NO.10,PP.5319-5325.

7. YURI BOYKOV, GARETH FUNKA -LEA" Graph cuts and efficien N-D image segmentation", International Computer Science, University of Western Ontario,London, ON Canada, April 2,2004. 